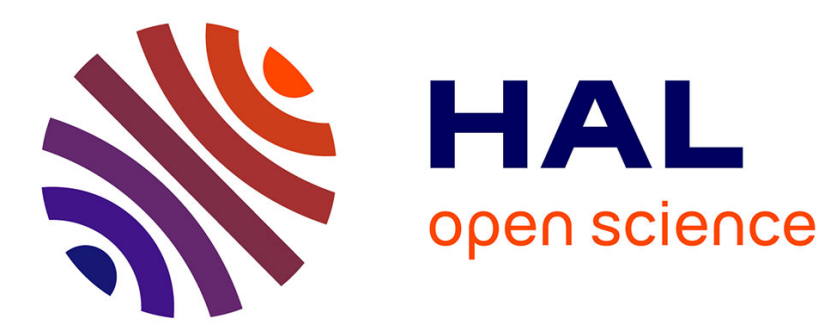

\title{
Une statue de Ptolémée Évergète Ier
}

Christophe Thiers

\section{To cite this version:}

Christophe Thiers. Une statue de Ptolémée Évergète Ier. Revue d'egyptologie, 1998, 49, pp.259-264. halshs-02051600

\section{HAL Id: halshs-02051600 https://shs.hal.science/halshs-02051600}

Submitted on 27 Feb 2019

HAL is a multi-disciplinary open access archive for the deposit and dissemination of scientific research documents, whether they are published or not. The documents may come from teaching and research institutions in France or abroad, or from public or private research centers.
L'archive ouverte pluridisciplinaire HAL, est destinée au dépôt et à la diffusion de documents scientifiques de niveau recherche, publiés ou non, émanant des établissements d'enseignement et de recherche français ou étrangers, des laboratoires publics ou privés. 


\section{Une statue de Ptolémée Évergète I ${ }^{\text {er }}$}

Le Musée Rodin à Paris conserve dans ses réserves, sous le numéro d'inventaire Co. 1414 (ex. 79), un fragment de statue en calcaire haut de $85 \mathrm{~cm}$, large de $32 \mathrm{~cm}$ et profond de $39 \mathrm{~cm}^{1}$. Il est signalé dans le catalogue Rodin collectionneur au $\mathrm{n}^{\circ} 19^{2}$. La tête, les bras et la partie inférieure des jambes sont perdus. Le personnage, la jambe gauche en avant, porte le pagne-shendjyt aux plis réguliers, tenu par une ceinture dépourvue de décoration. Ses deux bras reposaient le long du corps.

Les restes de la partie supérieure du buste suggèrent la présence d'un pendentif autour du cou du roi. Un examen attentif permet d'envisager l'existence d'une figurine dont on observe encore quelques traces. Il pourrait s'agir de la déesse Maât (restes de la plume?), notifiant la qualité de juge suprême du roi ${ }^{3}$; Diodore (I, 75) nous précise que le grand juge (archidicaste) «portait au cou, suspendue à une chaînette d'or, une image en pierres précieuses, représentant Alytheia». KhonsouThot, «le maître de la justice sur le grand siège pour départager les deux compagnons» se présente avec «sa Maât attachée à son cou» ${ }^{4}$.

Le texte des trois colonnes du pilier dorsal, bien que fort partiellement conservé, livre un intéressant éloge royal. La première colonne présente le protocole de Ptolémée Évergète Ir qu'il est aisé de restituer à l'aide des nombreux parallèles 5 .

\section{Col. 1:}

[L'Horus, Celui dont les dieux et les hommes se sont réjouis lorsqu'il a saisi] la royauté de son père, Celui des Deux Déesses, Vaillant, [celui qui protège les dieux, mur d'enceinte] bienfaisant de l'Égypte, l'Horus [d'Or], Grand [de puissance, qui accomplit des bienfaits, maître] des fêtes-sed comme son père Ptah[-Ténen, souverain comme Rê, le roi de Haute et Basse-Égypte, Héritier des dieux Adelphes, élu de Rê, image vivante d'Amon, le Fils de Rê, Ptolémée vivant à jamais, aimé de Ptah...].

Col. 2:

[...] pour gouverner la part (= propriété) des deux Maîtres; conscient alors qu'il était dans la matrice, (avant qu')il ne sortît de l'œuf, (le) Shaï s'est distingué sur sa (a) brique de naissance (b), élu du dieu qui ne fait qu'un avec l'infini (c), qui accroît son temps/année en centaine de milliers $[\ldots]$.

\footnotetext{
${ }^{1}$ Je remercie Mme Cl. Judrin, conservateur en chef au Musée Rodin, qui m'a autorisé à publier cette statue, ainsi que Mme B. Garnier pour sa disponibilité lors de l'examen de la statue. Mes remerciements s'adressent également à Ph. Collombert qui m'a signalé ce document et m'a transmis un premier relevé de l'inscription.

2 «Personnage masculin, dans l'attitude de la marche; au revers du pilier dorsal, trois colonnes d'inscriptions. Basse époque»; Rodin collectionneur, Musée Rodin, 1967-1968, nº 19.

3 B. Grdseloff, ASAE 40 (1940), p. 185-202; d'après Chr. Zivie, Giza au deuxième millénaire (BdE 70), 1976, p. 70, 1. 3 et p. 74, n. g; voir également B. Menu, EVO 17 (1994), p. 220-221. Cette figurine était une rpy.t (Edfou I, 580, 3); P. Wilson, A Ptolemaic Lexikon (OLA 78), 1997, p. 581.

${ }^{4}$ P. Clère, La Porte d'Évergète à Karnak (MIFAO 84), 1961, pl. 22 = Urk. VIII, 69-70 (83); d'après Ph. Derchain, dans Selbstverständnis und Realität. Akten des Symposiums zur ägyptischen Königsideologie in Mainz 15.-17.6.1995 (ÄAT 36, 1), 1997, p. 230.

${ }^{5}$ GLR IV, p. 254-257; D. Kurth, LÄ IV, col. 1194, s. v. Ptolemaios III. Evergetes.
} 


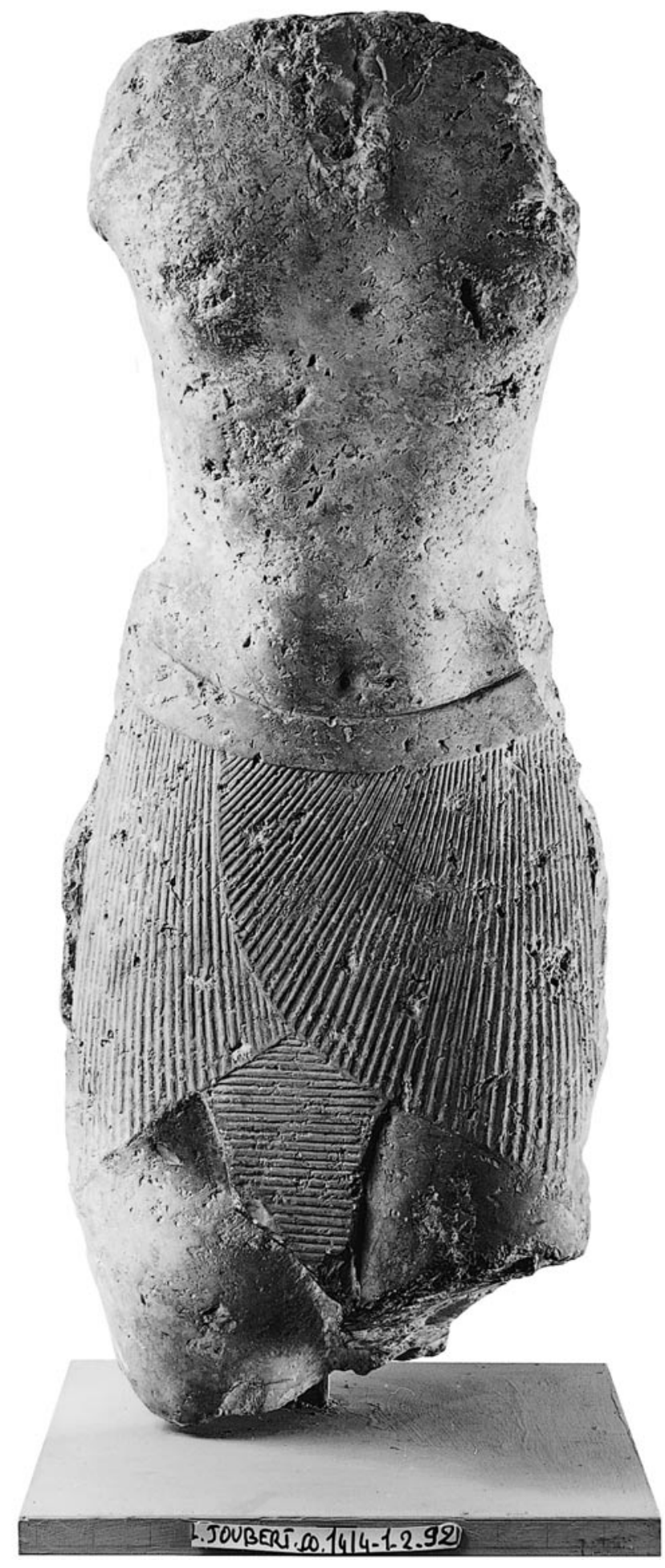

Collection des Antiques du Musée Rodin, Paris, inv. Co. 1414.

(c) musée Rodin (photo Luc et Lala Joubert). 


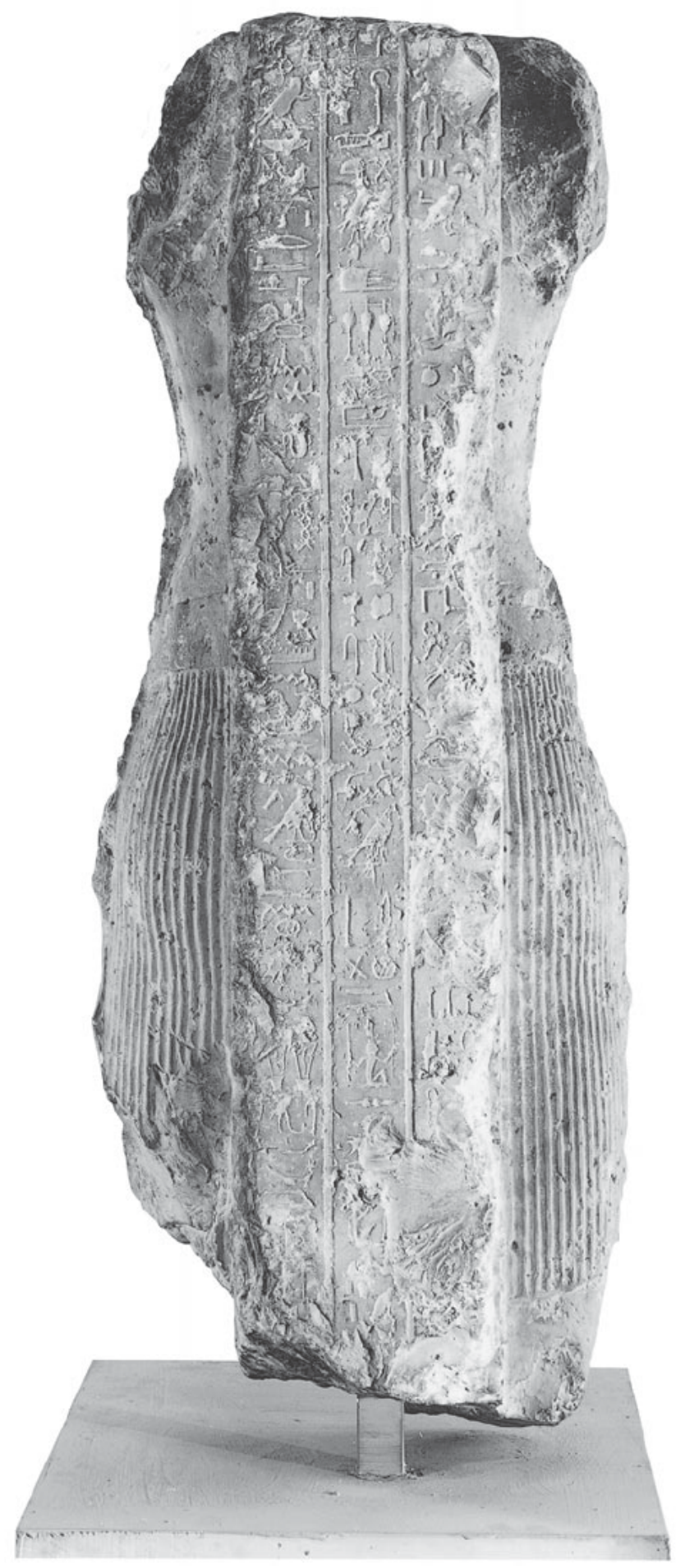

Collection des Antiques du Musée Rodin, Paris, inv. Co. 1414.

(c) musée Rodin (photo Luc et Lala Joubert). 


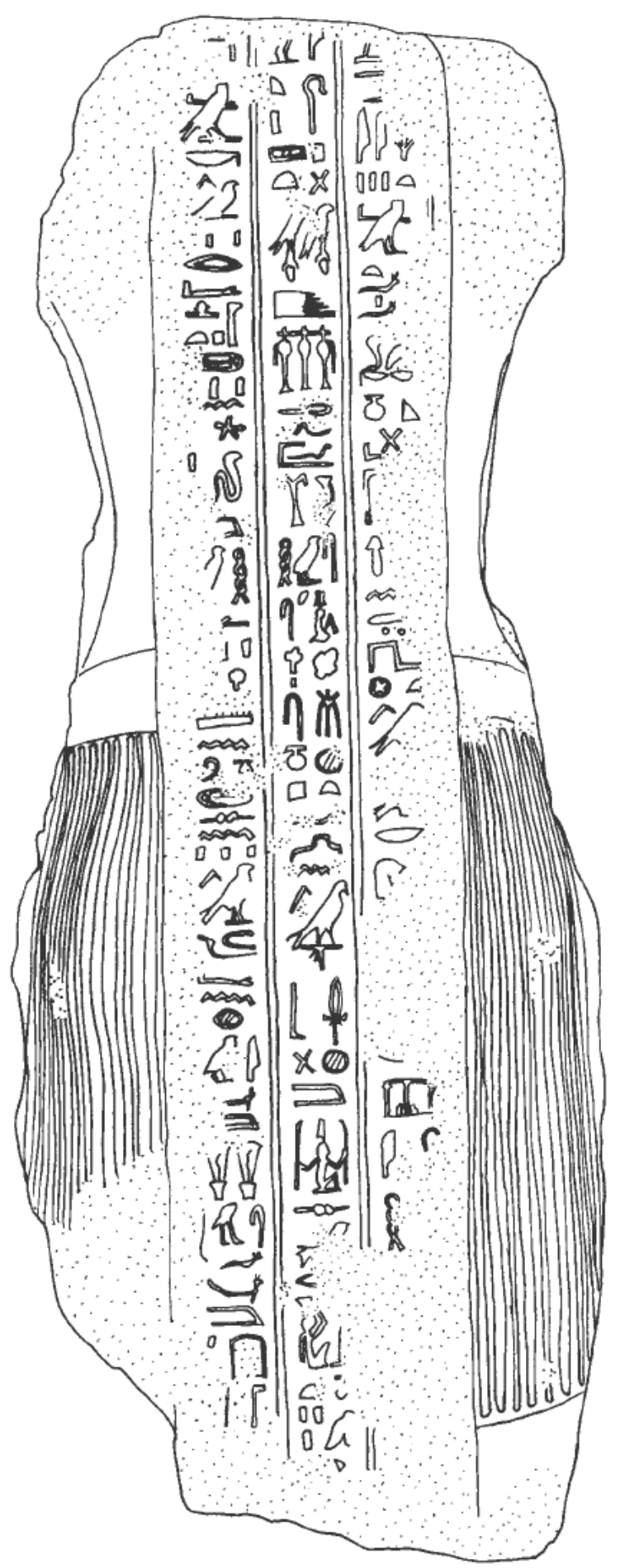

Musée Rodin Co. 1414. 
Col. 3:

[...] qui protège les images divines, qui dispense des offrandes divines aux dieux et aux déesses en plus de leurs offrandes quotidiennes (d), Horus vaillant, protecteur dans les sanctuaires, dont la statue est consacrée lors [des fêtes...] (e)».

(a) Le $=f$ est très dégradé mais sa lecture est sûre.

(b) Sur cette séquence mettant en exergue la prédestination royale, voir J. Quaegebeur, Le dieu Égyptien Shaï dans la religion et l'onomastique (OLA 2), 1975, p. 114-115, dont nous adoptons la traduction ${ }^{6}$.

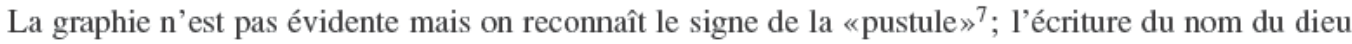
Shaï à l'aide de ce seul signe fait partie des «graphies exceptionnelles», selon l'inventaire dressé par J. Quaegebeur (o. c., p. 277, L 3). Cet auteur a également montré que ce dieu apparaissait en relation avec les souverains lagides à partir du règne d'Évergète I ${ }^{\text {er }}$ (ibid., p. 111) ${ }^{8}$. La séquence sj3 hnty h.t $n$ pr $=f m$ swh.t thj $s(w)$ š3w hr mshn.t se retrouve en Edfou $\mathrm{V}, 144,16-17 ;$ Kom Ombo $\mathrm{n}^{\circ}$ 603; Opet I, 264, 7; Esna II, n ${ }^{\circ} 150$.

(c) «Le dieu» apparaît ailleurs en relation avec Shaï et Renenet; c'est de lui que dépend la destinée du roi, destinée qui est ensuite prise en charge par Shaï et Renenet ${ }^{9}$. L'épithète «uni avec l'éternité» insiste sur l'intemporalité de ce dieu qui a également le pouvoir d'accrôtre le temps de vie du roi.

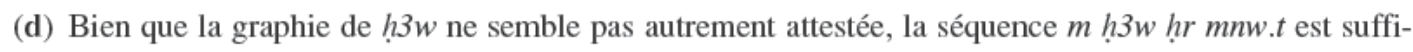
samment commune pour devoir être lue ici.

(e) $S s ̌ m=f h r p(w) m h h b . w$ (?) [...]; la partie supérieure du signe qui suit $m$ étant légèrement arrondie, une lecture $h b . w$ est préférable à une lecture $h w . w t$ (ou une précision topographique correspondant au temple de la ville d'où provenait cette statue) qui dans le contexte aurait également été bienvenue. On se rappellera que les prêtres réunis à Canope décidèrent «qu'un jour de fête en l'honneur des dieux Évergètes vienne en complément tous les quatre ans et s'ajoute aux cinq jours complémentaires avant le nouvel an» ${ }^{10}$. Aurait-on une allusion à cette fête?

Nous avons donc une statue consacrée au culte du Lagide dont avaient en charge les prêtres réunis dans une $5^{\mathrm{e}}$ phylé instituée par le synode de Canope. Cette statue constitue un témoin privilégié de la statuaire royale du règne d'Évergète $\mathrm{I}^{\mathrm{er}}$ dont on ne connaît pas d'exemples datés avec certitude $^{11}$; on regrettera d'autant plus la perte de la tête qui nous prive de précieuses indications stylistiques.

${ }^{6}$ J. Quaegebeur (Le dieu Égyptien Shaï dans la religion et l'onomastique (OLA 2), 1975, p. 114, n. 4) fait référence à la statue du Musée Rodin comme exemple de la séquence étudiée.

${ }^{7}$ M. Pezin et Fr. Janot, BIFAO 95 (1995), p. 361-365.

${ }^{8}$ Attestation sur la porte d'Évergète à Karnak, P. Clère, o. c., pl. 6 (Randzeile royale) =Urk. VIII, 53 (64), 5 (corriger la copie).

9 J. Quaegebeur, o. c., p. 106-108.

10 Urk. II, 141, 7-8; A. Bernand, La prose sur pierre dans l'Égypte hellénistique 1, 1992, p. 30.

${ }^{11}$ H. Kyrieleis, Bildnisse der Ptolemäer (ArchFor 2), 1975, p. 31-42; pour ce qui est de la statuaire égyptienne/égyptisante, voir une tête de New Haven (Yale University Gallery 4.1.1953) en basalte (p. 41 et pl. 28 (1-3)) attribuée d'après une analyse stylistique. Statue de basalte d'attribution incertaine découverte à la Villa Hadriana en 1899 (Berlin 14764); Staatliche Museen zu Berlin Ägyptisches Museum und Papyrussammlung, 1991, p. 190 (113) = A. Roullet, The Egyptian and Egyptianizing Monuments from Imperial Rome (EPRO 20), 1972, p. 105, $\mathrm{n}^{\circ} 166$. Voir également le fragment du pilier dorsal d'une statue en calcaire provenant de Coptos (Manchester, University Museum 1752); PM V, p. 133 = W.M.Fl. Petrie, Koptos, 1896, pl. XXVI (3 A), p. 22. Base de statue en pierre noire provenant de Behbeit el-Hagar (Caire 
Résumé / Abstract

Publication d'une statue ptolémaïque conservée au Musée Rodin (Paris). L'inscription du pilier dorsal livre la titulature de Ptolémée Évergète Ier ainsi qu'un éloge insistant sur la prédestination royale.

Publication of a ptolemaic statue keeped in Rodin Museum (Paris). The text on the back pillar presents the royal protocol of Ptolemy Evergete I and an eulogy, dwelling on the royal predestination.

Christophe THIERS

779 , route de Clapiers

34170 CASTELNAU-LE-LEZ

JE 41439); PM IV, p. 42 = P. Gallo, BIFAO 90 (1990), p. 226-228 et pl. VIII. La dernière étude sur la statuaire ptolémaïque (J.A. Josephon, Egyptian Royal Sculpture of the Late Period, 400-246 B.C. (DAIK 30), 1997) n'inclut pas le règne d'Évergète $\mathrm{I}^{\mathrm{er}}$. 